Research Paper

\title{
Comparative Analysis of Global Gene Expression and Complement Components Levels in Umbilical Cord Blood from Preterm and Term Neonates: Implications for Significant Downregulation of Immune Response Pathways related to Prematurity
}

\author{
Dorota Gródecka-Szwajkiewicz ${ }^{1^{*}}$, Zofia Ulańczyk ${ }^{1^{*}}$, Edyta Zagrodnik ${ }^{1^{*}}$, Karolina Łuczkowska1, Dorota \\ Rogińska1 , Miłosz P. Kawaํㅣ Iwona Stecewicz', Krzysztof Safranow², Przemysław Ustianowski³, Sławomir \\ Szymański ${ }^{4}$ Bogusław Machaliński ${ }^{\boxplus}$ \\ 1. Department of General Pathology, Pomeranian Medical University in Szczecin, Szczecin, Poland \\ 2. Department of Biochemistry and Medical Chemistry, Pomeranian Medical University in Szczecin, Szczecin, Poland \\ 3. Department of Perinatology, Obstetrics and Gynecology, Pomeranian Medical University in Szczecin, Szczecin, Poland \\ 4. Department of Obstetrics and Pathology of Pregnancy, Pomeranian Medical University in Szczecin, Szczecin, Poland \\ *These authors equally contributed to the work. \\ $\bowtie$ Corresponding author: Bogusław Machaliński, MD, PhD, DSc, Department of General Pathology, Pomeranian Medical University, Al. Powstańców Wlkp. 72, \\ 70-111 Szczecin, Poland. Tel. +4891 4661546; Fax +4891 4661548; E-mail:machalin@pum.edu.pl
}

(c) The author(s). This is an open access article distributed under the terms of the Creative Commons Attribution License (https://creativecommons.org/licenses/by/4.0/). See http://ivyspring.com/terms for full terms and conditions.

Received: 2020.03.25; Accepted: 2020.06.03; Published: 2020.07.11

\begin{abstract}
Background: Preterm birth is the most frequent cause of neonatal death, but its aetiology remains unclear. It has been suggested that the imbalance of immunological mechanisms responsible for maintaining pregnancy is contributing to preterm birth pathogenesis. We aimed to investigate global gene expression and the levels of several complement system components in umbilical cord blood samples from preterm neonates and compare them to term newborns. We sought to examine how differentially expressed genes could affect various immune-related pathways that are believed to be crucial factors in preterm birth.

Material and methods: We enrolled 27 preterm infants ( $<37$ weeks GA) and 52 term infants $(>37$ weeks GA), from which umbilical cord blood samples were collected. From these samples, peripheral blood mononuclear cells were isolated and subsequent RNA isolation was performed. We used Affymetrix Human Gene 2.1 ST Array Strip for microarray experiment and DAVID resources for bioinformatics analysis of the obtained data. Concentrations of $\mathrm{C} 2, \mathrm{C} 3 \mathrm{a}, \mathrm{C} 5 / \mathrm{C5}$, $\mathrm{C} 9$, FactorD, Properdin were measured in umbilical cord blood plasma samples using multiplex fluorescent bead-based immunoassays using Luminex technology.

Results: The levels of $\mathrm{C} 3 \mathrm{a}$ and $\mathrm{C} 5 / 5 \mathrm{a}$ were significantly elevated in preterm neonates compared to term babies, whereas $C 9$ concentration was evidently increased in term infants. The expression of 250 genes was upregulated at least 2-fold and 3781 genes were downregulated at least 2-fold in preterm neonates in comparison with term infants. Functional annotation analysis revealed that in preterm infants in comparison to term babies there was a significant downregulation of genes encoding several Toll-like receptors, interleukins and genes involved in major signalling pathways (e.g. NF-KB, MAPK, TNF, Notch, JAK) and vital cellular processes (e.g. intracellular signal transduction, protein ubiquitination, protein transport, RNA splicing, DNA-templated transcription).

Conclusions: Preterm birth results in immediate and long-term complications. Our results indicate that infants born prematurely show significant differences in complement components concentration and a downregulation of over 3,000 genes, involved mainly in various immune-related pathways, including innate immune response, phagocytosis and TLR function, when compared to full-term babies. Further studies on larger cohorts are needed to elucidate the role of immunity in prematurity.
\end{abstract}

Key words: premature birth, complement system proteins, immunity, gene expression 


\section{Introduction}

Preterm birth (PTB), defined by the World Health Organization (WHO) as a delivery prior to 37 completed weeks of gestation, accounts for roughly $5-18 \%$ of all births worldwide (1). Although PTB rates vary across the globe, one thing is common: PTB remains the most frequent cause of neonatal death (2). Even if a preterm infant survives, prematurity-related complications are tremendous and include neonatal respiratory conditions, necrotizing enterocolitis, sepsis, neurological conditions, visual and hearing problems (3). The higher rates of long-term morbidity in prematurely born babies in comparison to term newborns are attributed to immaturity of preterm infant's organs. In fact, gestational age (GA) at delivery and the risk of neonatal morbidity and mortality are inversely related with each other (4). PTB has also been linked with complications later in life, e.g. higher rates of hospital admissions, poorer neurodevelopmental outcomes, behavioural and social difficulties (5). The medical impact and socio-economic burden of preterm birth consequences make better understanding and therefore possible prevention of PTB a public health priority.

However, PTB aetiology remains not fully understood. Despite the multitude of risk factors identified (6), the majority of preterm births do not have a specific risk factor. Several different pathological processes have been implicated in preterm birth, including ischemia, stress, infections, cervical diseases and other (7). One particular factor that has been increasingly implicated in PTB pathogenesis is dysregulation of a fine balance of immunological mechanisms normally responsible for maintaining pregnancy (8). The maternal immune system faces a considerable challenge during pregnancy, as it has to tolerate the paternal alloantigens expressed in foetal tissues and simultaneously maintain effective protection against microbes with innate and adaptive immunity mechanisms (9).

According to Gomez-Lopez et al., during late pregnancy, the pro-inflammatory nuclear factor-kB (NF-KB) signalling pathway is activated and that leads to recruitment of circulating maternal leukocytes at maternal/foetal interface by chemotaxis (10). Premature shift from anti-inflammatory to this pro-inflammatory state is believed to contribute to a loss in foetal-maternal tolerance and consequent labour initiation, leading to preterm delivery (11). The imbalance of both innate and adaptive immunity systems components have been implicated in PTB or miscarriage, but the role of immunity deficiencies in preterm infants seems to be even more profound (12). Numerous studies have shown that immune system of preterm neonates is more immature than that of term ones $(13,14)$. In fact, both innate and adaptive immunity of preterm babies is diminished, as are the interactions between these two systems. It has been also hypothesised that PTB may shape preterm infants' immune system via various common pre- and postnatal events associated with it that have the potential to influence immunity (15).

Neonates are forced to rely mainly on their innate immune defence, because their inexperienced adaptive immune system fully develops only later, in the early childhood (16). Preterm infants are more prone to bacterial infections than babies born at term and there are several reasons for that, including prolonged intensive care, but the immaturity of innate immune system remains a major cause (13). Term infants could be considered 'lucky' to have received maternal antibodies that serve as supplemental protection, whereas preterm infants 'missed' the largest part of transfer of maternal antibodies, as it increases with foetal age (17). As one of the most important constituents of innate immunity, the role of complement in PTB and prematurity has also been implicated (18). The maturity of complement components and their concentrations seems inadequate even in healthy term newborns, and these deficiencies are even more profound in preterm neonates (18). Complement activation is crucial in pathogen opsonization, anaphylatoxins synthesis, leukocyte recruitment and bacteria lysis (19). However, in preterm infants, complement activation pathways (classical, alternative, lectin) show profound dysregulation in their pathogen-killing abilities (20). Although the role of immunity in prematurity is getting more attention, its exact role in this condition and how differentially expressed genes could affect various immune-related pathways leading to PTB and/or affecting preterm newborns remains not fully understood.

In our previous studies, we have investigated umbilical cord blood (UCB) expression levels of small non-coding RNAs (miRNAs) potentially involved in angiogenesis control in preterm newborns and term neonates (21). In this study, we aimed to investigate global gene expression in UCB samples from preterm neonates and compare it to term newborns. In addition, we sought to examine how these differentially expressed genes could affect various immune-related pathways that are said to be contributing to preterm birth. Moreover, we aimed to compare the levels of several crucial components of complement system in UCB plasma samples between preterm and term infants. 


\section{Methods}

\section{Study groups characteristics}

In total, 27 preterm infants born $<37$ weeks GA and 52 term infants born at $>37$ weeks GA all appropriate-for-gestational-age were enrolled in this study from the Department of Obstetrics and Gynaecology of the Pomeranian Medical University in Szczecin, Poland. We excluded subjects with severe congenital malformations, known chromosomal abnormalities, intracranial haemorrhage, cyanotic heart defects, inherited metabolic disorders, severe anaemia, congenital infections and severe infectious diseases, maternal history of tobacco and/or alcohol abuse; maternal infections were also excluded. For each recruited neonate we documented sex, GA, birth weight and physical measurements, Apgar score, clinical course. The study adhered to the tenets of the Declaration of Helsinki, and approval was obtained from the Local Ethics Committee. All parents provided written informed consent for their children participation in the study.

\section{Umbilical cord blood collection}

We collected autologous umbilical cord blood from all patients at birth in accordance with NetCord-FACT International Standards for Cord Blood Collection (22). Collections were performed in utero as described previously (23), using a collection bag system with citrate-phosphate-dextrose (CPD) as anticoagulant. UCB samples were then centrifuged (2000 $\mathrm{rpm}, 4^{\circ} \mathrm{C}, 10 \mathrm{~min}$ ), and the plasma was removed and stored $\left(-20^{\circ} \mathrm{C}\right.$ to $\left.-80^{\circ} \mathrm{C}\right)$ until further assayed. Next, we used BD Pharm Lyse lysing buffer (BD Biosciences, San Jose, CA, United States) for red blood cells lysis to obtain peripheral blood mononuclear cells (PBMCs).

\section{Luminex assay}

Concentrations of $\mathrm{C} 2, \mathrm{C} 3 \mathrm{a}, \mathrm{C} 5 / \mathrm{C} 5 \mathrm{a}, \mathrm{C} 9$, FactorD, Properdin were measured in UCB plasma using multiplex fluorescent bead-based immunoassays (Luminex Corporation, Austin, TX, USA). The procedure was performed according to the manufacturer's protocol, as previously described (24). In brief, $50 \mu \mathrm{L}$ of blank, standards and samples were added to the plate together with Microparticle Cocktail. This was followed by incubation step for 2 hours in the dark at room temperature on horizontal orbital microplate shaker (800rpm). Then, $100 \mu \mathrm{L}$ of wash buffer was used to was the wells three times. Next, $50 \mu \mathrm{L}$ of biotin-antibody cocktail was added to the plate and incubated for 1 hour in the dark. After subsequent washing step, Streptavidin-PE was added $(50 \mu \mathrm{L})$ to each well and incubated in the dark for 30 .
After washing, the microspheres in each well were resuspended in wash buffer $(100 \mu \mathrm{L})$ and shaken for 2 minutes. Finally, the plate was read on the Luminex 200 analyzer. The tested proteins concentrations were calculated from seven different standard curves that present median fluorescence intensity vs protein concentration.

\section{RNA isolation}

The mirVana ${ }^{\mathrm{TM}}$ miRNA Isolation Kit (Thermo Fisher, Waltham, MA, USA) was used for total RNA isolation from collected umbilical cord blood cells $\left(1 \times 10^{6}\right)$. Concentration and quality of the obtained RNA was assessed using Epoch spectrophotometer (Biotek, Winooski, VT, USA).

\section{Affymetrix GeneChip Microarray and Data Analysis}

Total RNA isolated from UCB samples was pooled to generate one sample per group for microarray experiment. The procedure was performed according to the manufacturer's protocol, as previously described (21). Generation of sense strand cDNA from the total RNA and subsequent fragmentation and labelling steps were made using GeneChip ${ }^{\mathrm{TM}}$ WT PLUS Reagent Kit (Thermo Fisher Scientific, Waltham, MA, USA). Finally, the sample was hybridized onto an Affymetrix Human Gene 2.1 ST Array Strip. Affymetrix GeneAtlas System was used for hybridization, fluidics and scanning steps. Subsequent analyses were performed using BioConductor software. We used Robust Multiarray Average (RMA) normalisation algorithm (from "Affy" library) for normalisation, background correction, and calculation of the expression levels of examined genes.

\section{DAVID}

For functional annotation and enrichment analysis, we used DAVID Bioinformatics Resources (Database for Annotation, Visualization, and Integrated Discovery) at http://david.abcc.ncifcrf. gov, as previously described $(25,26)$. Functional annotation charts generated by DAVID with overrepresented gene annotations are shown as bubble plots from BACA BioConductor package (https://cran.r-project.org/web/packages/BACA/B ACA.pdf). The following criteria were applied to generate bubble plots: $p$-value $<0.5$, adjusted method $=$ Benjamini (27), and minimal number of genes per group $=5$. Groups of genes that meet this criteria are shown in a graph, where the bubble size is indicative of the number of genes represented in the corresponding annotation and their down- or up-regulation. 


\section{Statistical analysis}

The nonparametric Mann-Whitney test was used to compare values between study groups, because in majority of cases the quantitative variables distribution notably differed from the normal distribution. Spearman's rank correlation coefficient (Rs) was used to measure the strength of associations between gestational age and concentrations of each tested complement component. The sign of the Rs value indicates the direction of the association (positive or negative), while higher absolute value (closer to -1 or +1 ) indicates stronger association. We considered $p<0.05$ statistically significant. Statistica 13 software (Dell Inc., OK, USA) was used for statistical analysis.

\section{Results}

\section{Study Groups Characteristics}

In total, 27 preterm and 52 term neonates were enrolled in this study. The characteristics of the study groups are summarized in Table 1 . The preterm neonates differed from term neonates in most of the recorded measurements, including Apgar scores, arterial-blood gas test results, blood cell counts and physical measurements. The groups did not differ in CRP and IL-6 concentrations, indicating lack of apparent acute inflammation and potential pro-inflammatory status of both groups.

\section{UCB plasma complement component levels}

From six factors tested, we observed differences in concentration of $\mathrm{C} 3 \mathrm{a}, \mathrm{C} 5 / \mathrm{C} 5 \mathrm{a}$ and $\mathrm{C} 9$ between the study groups (Figure 1). The mean concentrations of $\mathrm{C} 3 \mathrm{a}$ and C5/5a were significantly elevated in preterm neonates comparing to term babies $(\mathrm{p}<0.001$ and $\mathrm{p}<0.01$, respectively). In contrast, C9 concentration was evidently increased $(\mathrm{p}<0.01)$ in term infants when compared with preterm neonates. The rest of the tested factors concentrations did not differ between preterm and term infants.

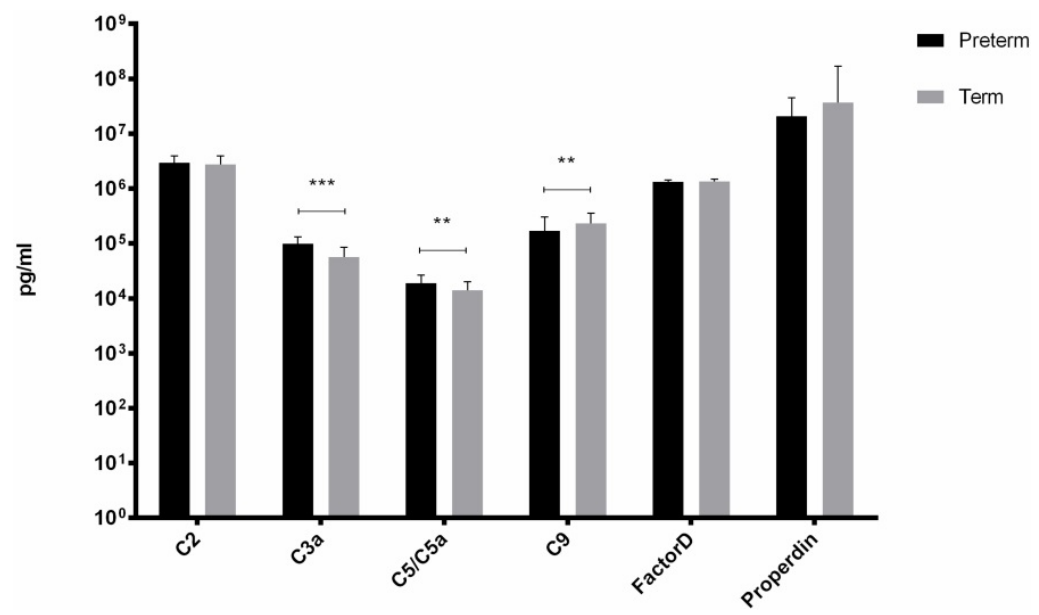

Figure 1. Complement components levels in UCB from preterm and term infants ( $* *$ p-value $<0.01$; *** p-value $<0.001$ ).

Table 1. Characteristics of the study groups. In bold, $p$-value $<0.05$ considered statistically significant.

\begin{tabular}{|c|c|c|c|c|c|c|c|c|c|}
\hline Parameter & Preterm infar & & & & Term infants & & & & \\
\hline Number of subjects & 27 & & & & 52 & & & & \\
\hline $\operatorname{Sex}(M / F)$ & $15 / 12$ & & & & $32 / 20$ & & & & \\
\hline Parameter & Mean $\pm S D$ & Median & Q1 & Q3 & Mean \pm SD & Median & Q1 & Q3 & p-value \\
\hline Gestational age [weeks] & $33.0 \pm 2.6$ & 38.5 & 38 & 39 & $38.4 \pm 1.0$ & 33 & 32 & 35 & $<0.001$ \\
\hline Weight $[\mathrm{g}]$ & $2114.1 \pm 648.7$ & 2010 & 1700 & 2700 & $3171.0 \pm 520.7$ & 3220 & 2770 & 3500 & $<0.001$ \\
\hline Length $[\mathrm{cm}]$ & $46.0 \pm 5.7$ & 46 & 42 & 50 & $53.5 \pm 3.5$ & 54 & 51 & 56 & $<0.001$ \\
\hline Chest circumference $[\mathrm{cm}]$ & $27.4 \pm 3.3$ & 27 & 26 & 30 & $32.6 \pm 2.3$ & 33 & 31 & 34 & $<0.001$ \\
\hline Head circumference $[\mathrm{cm}]$ & $29.9 \pm 2.7$ & 30 & 28 & 32 & $34.1 \pm 1.8$ & 34 & 33 & 35 & $<0.001$ \\
\hline Apgar score at $1 \mathrm{~min}$ & $8.2 \pm 1.8$ & 8 & 8 & 10 & $9.3 \pm 1.0$ & 10 & 9 & 10 & 0.001 \\
\hline Apgar score at $5 \mathrm{~min}$ & $8.9 \pm 1.2$ & 9 & 8 & 10 & $9.7 \pm 0.6$ & 10 & 9 & 10 & $<0.001$ \\
\hline $\mathrm{pO}_{2}[\mathrm{mmHg}]$ & $25.2 \pm 12.6$ & 21.8 & 17.6 & 27 & $19.4 \pm 13.7$ & 14.15 & 12.4 & 20.6 & 0.002 \\
\hline $\mathrm{pCO}_{2}[\mathrm{mmHg}]$ & $46.1 \pm 6.7$ & 44.3 & 41.3 & 50.5 & $50.0 \pm 8.1$ & 49.95 & 45.7 & 53.3 & 0.02 \\
\hline $\mathrm{pH}$ & $7.32 \pm 0.07$ & 7.32 & 7.28 & 7.35 & $7.32 \pm 0.05$ & 7.32 & 7.29 & 7.34 & 0.85 \\
\hline Haemoglobin $[\mathrm{g} / \mathrm{dL}]$ & $16.0 \pm 1.7$ & 16.2 & 14.6 & 17.2 & $16.8 \pm 3.7$ & 17.3 & 14.9 & 19.3 & 0.06 \\
\hline RBC count $\left[10^{6} / \mu \mathrm{L}\right]$ & $4.3 \pm 0.5$ & 4.3 & 4.0 & 4.7 & $4.9 \pm 0.8$ & 4.8 & 4.4 & 5.3 & 0.001 \\
\hline Platelets $\left[10^{3} / \mu \mathrm{L}\right]$ & $268.3 \pm 62.0$ & 269 & 239 & 294 & $226.5 \pm 68.0$ & 237 & 198 & 275 & 0.01 \\
\hline WBC count $\left[10^{3} / \mu \mathrm{L}\right]$ & $12.5 \pm 5.1$ & 12 & 16.8 & 8.7 & $19.8 \pm 7.8$ & 20 & 15 & 23.8 & $<0.001$ \\
\hline $\mathrm{CRP}[\mathrm{mg} / \mathrm{dL}]$ & $1.7 \pm 1.8$ & 1 & 1 & 1 & $2.0 \pm 2.4$ & 1 & 0.8 & 2.6 & 0.92 \\
\hline IL-6 [pg/mL] & $109.3 \pm 184.0$ & 29 & 11 & 58 & $19.1 \pm 17.6$ & 16.8 & 3.5 & 21 & 0.07 \\
\hline
\end{tabular}


Table 2. The list of 10 most upregulated genes in PBMCs isolated from UCB samples from preterm neonates in comparison with term infants.

\begin{tabular}{|c|c|c|c|}
\hline Gene symbol & Gene name & Entrez Gene ID & Fold change \\
\hline RUNDC3A & RUN domain containing 3A & 10900 & 18.73 \\
\hline SPTA1 & spectrin, alpha, erythrocytic 1 & 6708 & 17.98 \\
\hline HLA-DRB5 & major histocompatibility complex, class II, DR beta 5 & 3127 & 17.24 \\
\hline DDX3Y & DEAD (Asp-Glu-Ala-Asp) box helicase 3, Y-linked & 8653 & 13.42 \\
\hline FHDC1 & FH2 domain containing 1 & 85462 & 12.73 \\
\hline SLC4A1 & solute carrier family 4 (anion exchanger), member 1 (Diego blood group) & 6521 & 10.35 \\
\hline HLA-DRB1 & major histocompatibility complex, class II, DR beta 1 & 3123 & 9.97 \\
\hline EPB42 & erythrocyte membrane protein band 4.2 & 2038 & 9.42 \\
\hline SLC2A1 & solute carrier family 2 (facilitated glucose transporter), member 1 & 6513 & 8.46 \\
\hline HBG1 & hemoglobin, gamma A & 3047 & 7.59 \\
\hline
\end{tabular}

Table 3. The list of 10 most downregulated genes in PBMCs isolated from UCB samples from preterm neonates in comparison with term infants.

\begin{tabular}{|c|c|c|c|}
\hline Gene symbol & Gene name & Entrez Gene ID & Fold change \\
\hline CYP4F3 & cytochrome P450, family 4, subfamily F, polypeptide 3 & 4051 & -14.55 \\
\hline CEP19 & centrosomal protein $19 \mathrm{kDa}$ & 84984 & -14.18 \\
\hline FFAR2 & free fatty acid receptor 2 & 2867 & -13.86 \\
\hline NUAK2 & NUAK family, SNF1-like kinase, 2 & 81788 & -12.83 \\
\hline TRBV20-1 & $\mathrm{T}$ cell receptor beta variable $20-1$ & --- & -12.50 \\
\hline FRAT2 & frequently rearranged in advanced T-cell lymphomas 2 & 23401 & -12.45 \\
\hline TNFAIP6 & tumor necrosis factor, alpha-induced protein 6 & 7130 & -12.18 \\
\hline SNORD84 & small nucleolar RNA, C/D box 84 & 692199 & -11.99 \\
\hline PI3 & peptidase inhibitor 3, skin-derived & 5266 & -11.43 \\
\hline S1PR4 & sphingosine-1-phosphate receptor 4 & 8698 & -11.25 \\
\hline
\end{tabular}

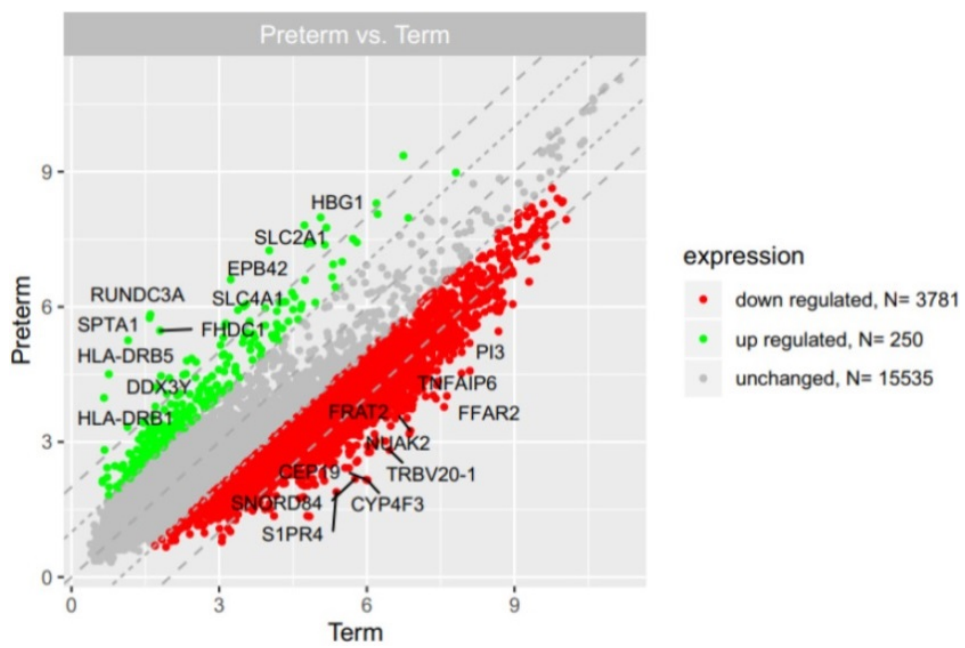

Figure 2. The scatter plot of global gene expression in UCB from preterm neonates when compared to term ones. Red points correspond to downregulated genes (at least 2 -fold change, $p<0.05$ ), green points show upregulated genes (at least 2 -fold change, $p<0.05$ ). The graph also contains names of the genes with the highest change in expression.

There was a significant positive correlation between GA and C5/C5a (Rs $=+0.35, p=0.01)$ and C9 $(R s=+0.45, p<0.001)$ in term infants and between GA and C2 $(\mathrm{Rs}=+0.45, \mathrm{p}=0.02)$ in preterm infants.

\section{Gene expression profile in PBMCs isolated from UCB samples}

Microarray analysis showed that expression of 250 genes was upregulated at least 2-fold (fold 18.73 to 2) and 3781 genes were downregulated at least 2-fold (fold -14.55 to -2) in PBMCs isolated from UCB samples from preterm neonates in comparison with term infants (Figure 2). The list of 10 genes with the most significantly up- and downregulated expression is presented in Table 2 and Table 3 , respectively.

Next, differentially expressed genes were classified according to the Gene Ontology (GO) Classification of biological processes and KEGG Database of pathways. Analysis of functional annotations mainly identified downregulated processes (e.g. signal transduction, DNA transcription, innate immune response) in PBMCs from UCB of preterm neonates when compared with term infants. The results of these analyses are displayed as separate bubble graphs for biological processes and pathways (Figure 3 and 4, respectively). 


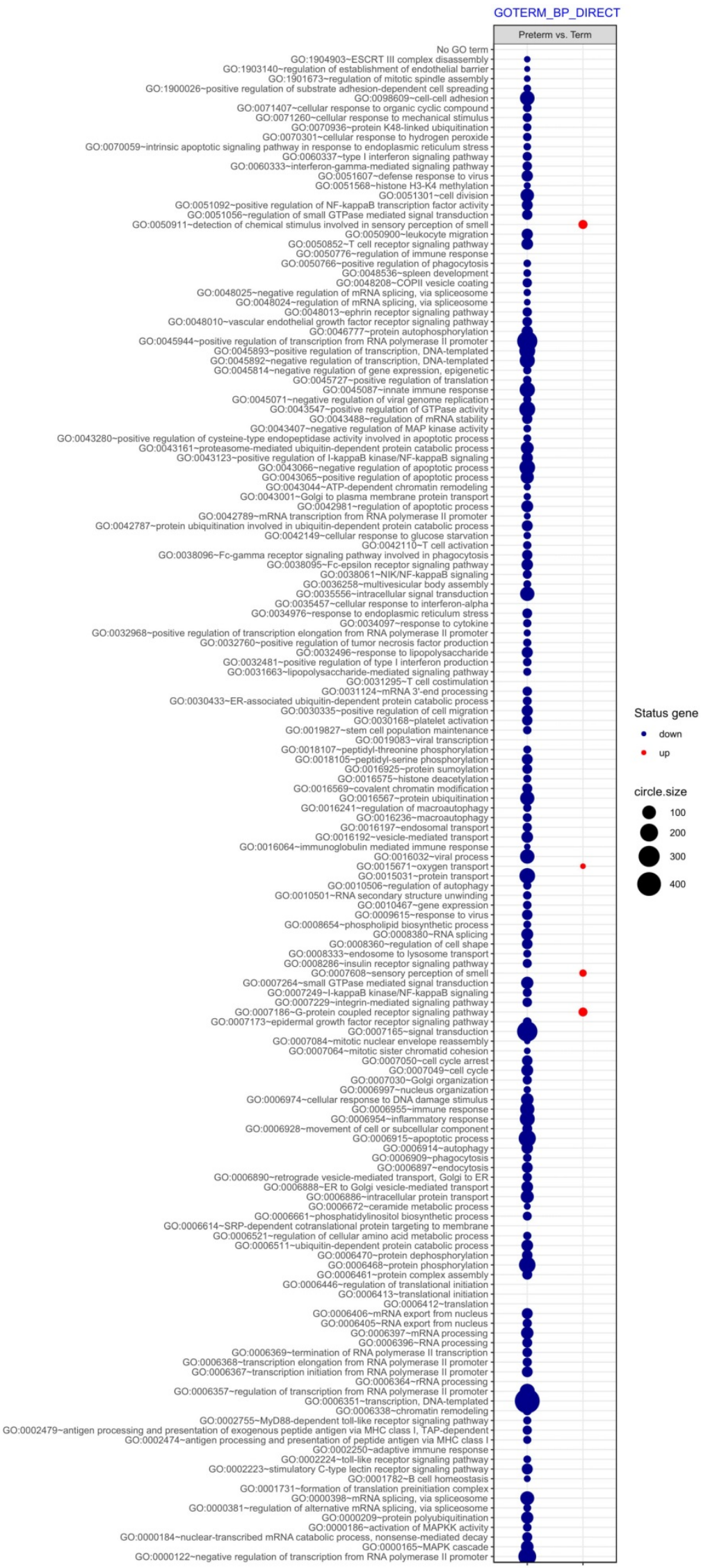

Figure 3. The bubble plot of overrepresented biological processes assigned according to Gene Ontology (GO) classification in PBMCs isolated from UCB samples from preterm neonates in comparison with term infants. Following criteria were applied to assign genes included in the graph to individual processes: adjusted $p<0.05$, method $=$ Benjamini, minimum number of genes per group $=5$. The size of each bubble indicates the number of genes represented in the corresponding annotation and the colour reflects the status of genes in terms of their up- or downregulation. 


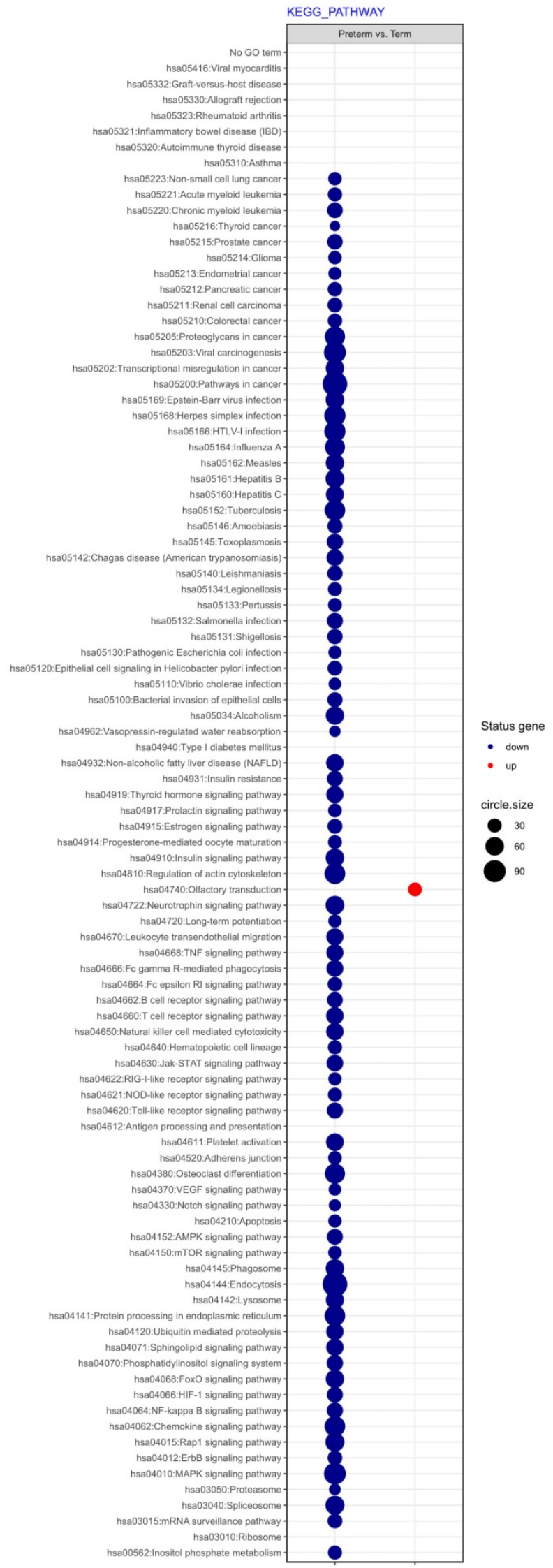

Figure 4. The bubble plot of changed pathways assigned according to KEGG Pathway Database in PBMCs isolated from UCB samples from preterm neonates in comparison with term infants. Following criteria were applied to assign genes included in the graph to individual processes: adjusted $p<0.05$, method $=$ Benjamini, minimum number of genes per group $=5$. The bubble size reflects the number of genes represented in the corresponding annotation and the colour reflects the status of genes in terms of their up- or downregulation. 


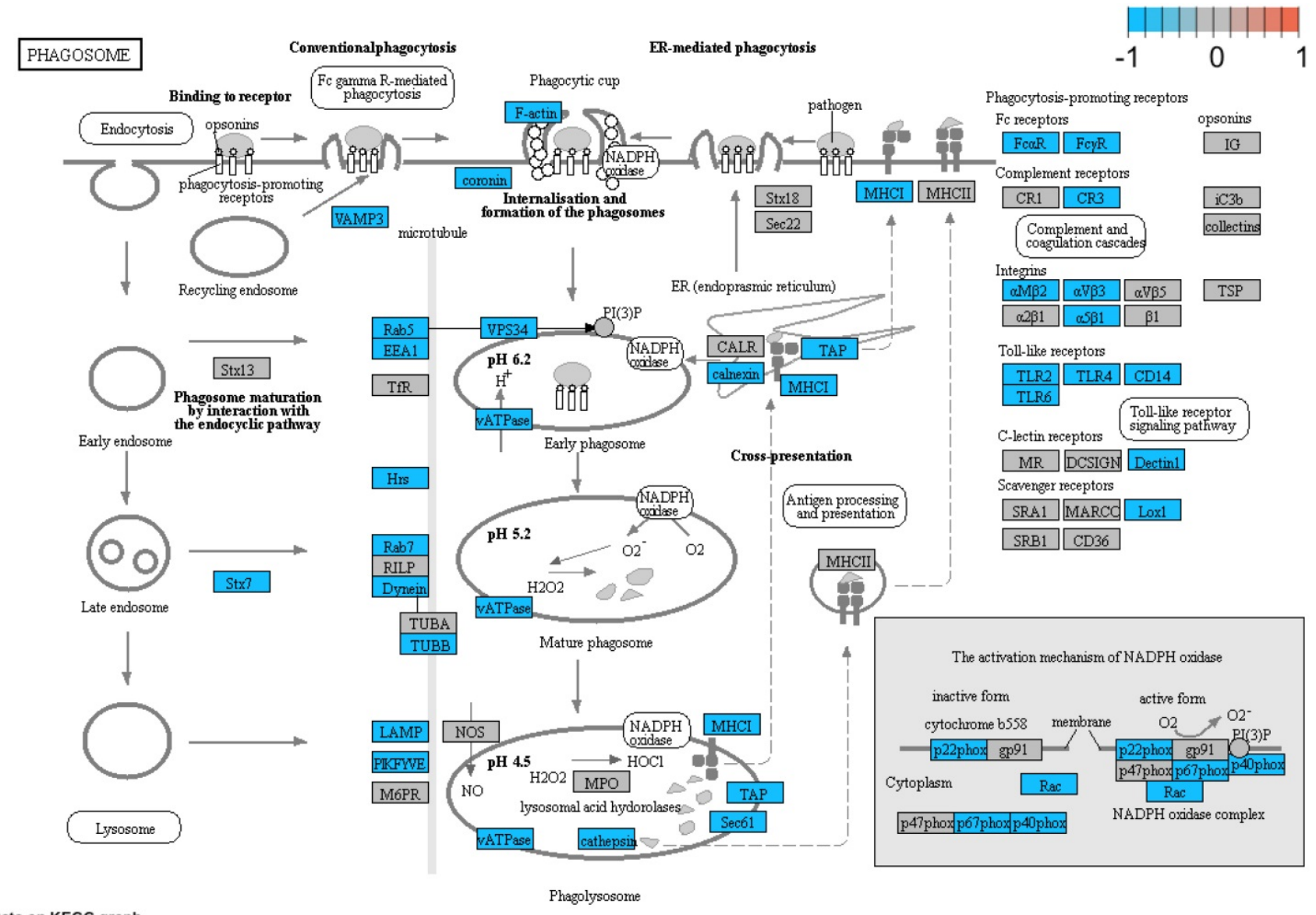

Data on KEGG graph

Rendered by Pathview

Figure 5. Genes and processes involved in the 04145.Phagosome pathway. The colour scale corresponds to log(FC) value, where FC stands for gene expression fold change when comparing samples from preterm to term neonates.

Additionally, we performed a detailed analysis of three signalling pathways (Figure 5: 04145.Phagosome; Figure 6: 04660.T cell receptor signalling pathway; Figure 7: 04662.B cell receptor signalling pathway), in order to better understand the effects of specific genes dysregulation on the selected process. The graphs present genes (and their up- or downregulation) and relationships between them. The majority of genes involved in these processes were significantly downregulated in samples from preterm neonates when compared to full-term infants, including genes encoding Toll-like receptors, Fc receptors, integrins, TNF- $\alpha$ and also genes encoding proteins involved in MAPK, NF-KB, PI3K-Akt and calcium signalling pathways.

Next, differentially expressed genes were classified according to the Gene Ontology (GO) Classification of biological processes and KEGG Database of pathways. Analysis of functional annotations mainly identified downregulated processes (e.g. signal transduction, DNA transcription, innate immune response) in PBMCs from UCB of preterm neonates when compared with term infants. The results of these analyses are displayed as separate bubble graphs for biological processes and pathways (Figure 3 and 4, respectively). Additionally, we performed a detailed analysis of three signalling pathways (Figure 5: 04145.Phagosome; Figure 6: 04660.T cell receptor signalling pathway; Figure 7: 04662.B cell receptor signalling pathway), in order to better understand the effects of specific genes dysregulation on the selected process. The graphs present genes (and their up- or downregulation) and relationships between them. The majority of genes involved in these processes were significantly downregulated in samples from preterm neonates when compared to full-term infants, including genes encoding Toll-like receptors, Fc receptors, integrins, TNF- $\alpha$ and also genes encoding proteins involved in MAPK, NF-kB, PI3K-Akt and calcium signalling pathways.

Finally, we chose three significantly downregulated immune-related processes in preterm neonates (innate immune response, immune response, phagocytosis) and mapped the relationships between genes and GO terms in these processes with circos plots with visualization of FC (fold change) values and gene symbols (Figure 8). 


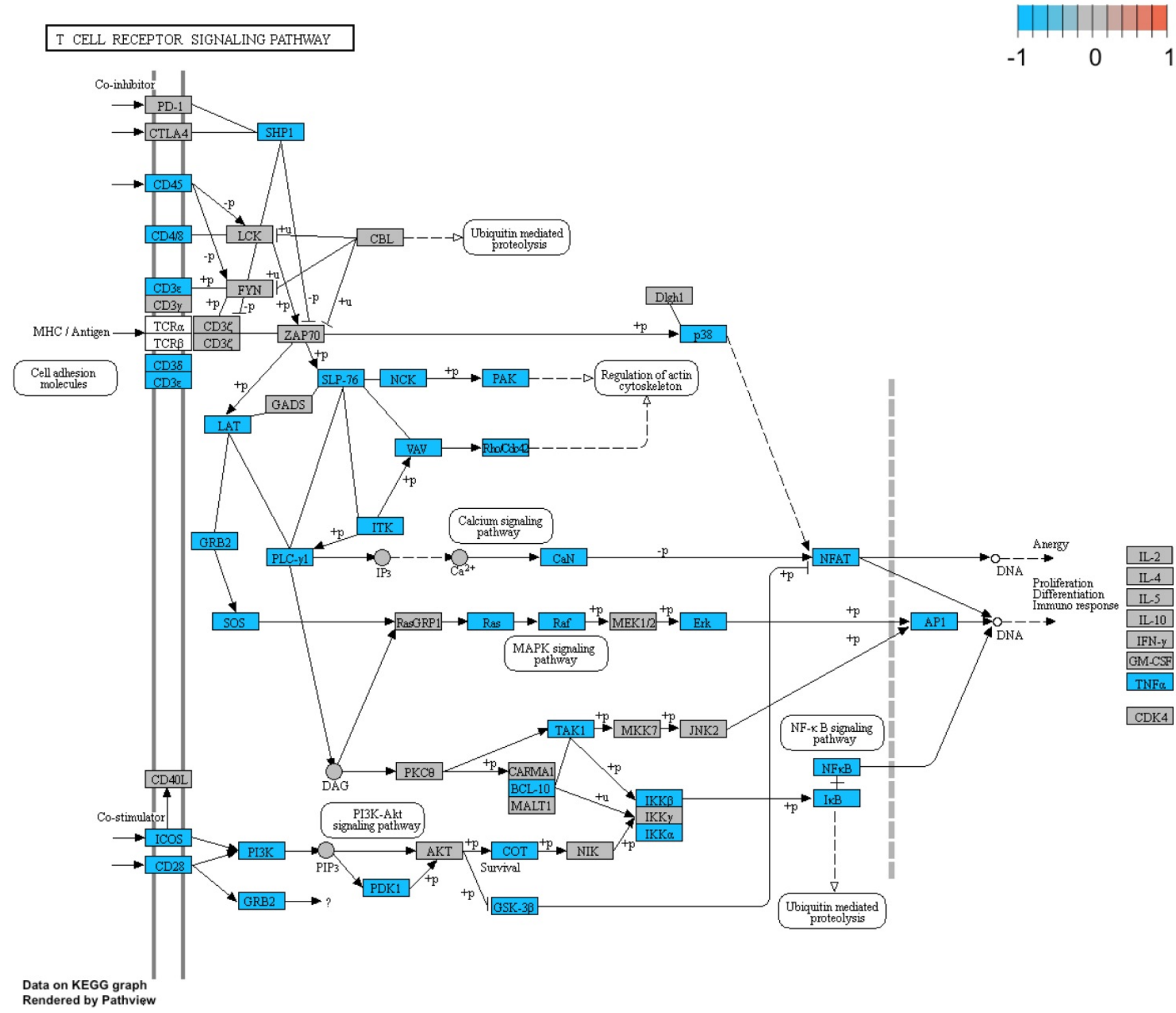

Figure 6. Genes and processes involved in the 04660.T cell receptor signalling pathway. The colour scale corresponds to log(FC) value, where FC stands for gene expression fold change when comparing samples from preterm to term neonates.

Overall, the obtained results indicate significant downregulation of genes involved in immune response and related signalling pathways (e.g. phagocytosis, immunoglobulin mediated immune response, inflammatory response) in preterm infants when compared to term neonates. In particular, the expression of genes encoding several Toll-like receptors (TLRs) family members (TLR1, 2, 4, 5, 6, 8 and TLR10), and of various genes associated with interleukins (e.g. IL1RAP, IL18R1, ILF2, IL7R, IL10RB) was decreased in PBMCs from UCB samples from preterm neonates. In addition, major signalling pathways (e.g. NF-kB, MAPK, TNF, Notch, JAK) and vital cellular processes (e.g. intracellular signal transduction, protein ubiquitination, protein transport, RNA splicing, DNA-templated transcription) were also significantly downregulated in preterm neonates in comparison with term infants.

\section{Discussion}

Preterm birth remains the leading cause of neonatal death and both short- and long-term consequences of prematurity are detrimental. The pathogenesis of PTB remains mostly unknown, though immune-related processes have been postulated to play a significant role in it. In this study we compared the levels of complement system components and global gene expression in UCB samples between preterm and term infants. We found that levels of complement components $\mathrm{C} 3 \mathrm{a}$ and $\mathrm{C} 5 \mathrm{a}$ were significantly elevated in preterm babies, whereas expression of over 3,000 genes, multitude of them associated with immunity, was downregulated in those neonates in comparison with term newborns.

Foetal complement components are synthesized mainly in the liver and are well detectable around 
18-20 weeks of gestation (28). Although increased complement activity in maternal circulation has been linked with PTB and elevated complement components in amniotic fluid are indicative of intrauterine infection, little or no maternal complement components are transferred to the foetus $(8,28)$. Our finding of elevated proinflammatory anaphylotoxins, C3a and C5a, in preterm infants is in contrary with previous studies, which in majority show that prematurity is rather related to lower levels of complement components $(29,30)$. Early studies from the 1970s-80s showed that the overall functional output of the classical complement pathway and the levels of the components C3, C4 and C5 were lower in preterm infants than in term neonates, whereas term neonates presented with comparatively low levels of those components in comparison with adults $(29,31)$. These complement deficiencies in preterm neonates are thought to be responsible for delayed inflammatory responses and impaired bacterial defences, leading to increased susceptibility to infections (32, 33). However, considerable interindividual variability in complement components concentration in neonates has been observed (34). On the other hand, the increase in anaphylotoxins in our preterm group could be attributed to the crosstalk between coagulation and complement cascades. Certain coagulation system components, such as thrombin and factor $\mathrm{Xa}$, can activate C3 and C5 independently of the established complement activation pathways and these anaphylotoxins may in turn intensify coagulation (18, 35). Therefore, given that prematurity is associated with various vascular events like thrombosis and ischemia, we cannot exclude that the observed increase in anaphylotoxins levels is due to intensified coagulation and thus activation of complement cascade components.

It has also been indicated that increase in plasma complement components in apparently infection-free premature neonates could be either due to subclinical infection (enhanced activation of complement) and/or lower binding potential of complement receptors $(36,37)$. We cannot exclude that there were subjects with ongoing infection in our preterm group at very subclinical stage as concentrations of both CRP and IL-6 were not significantly changed in examined groups, nor can we reject the hypothesis of diminished receptor binding potential since complement component 5a receptor 1 gene (C5AR1)

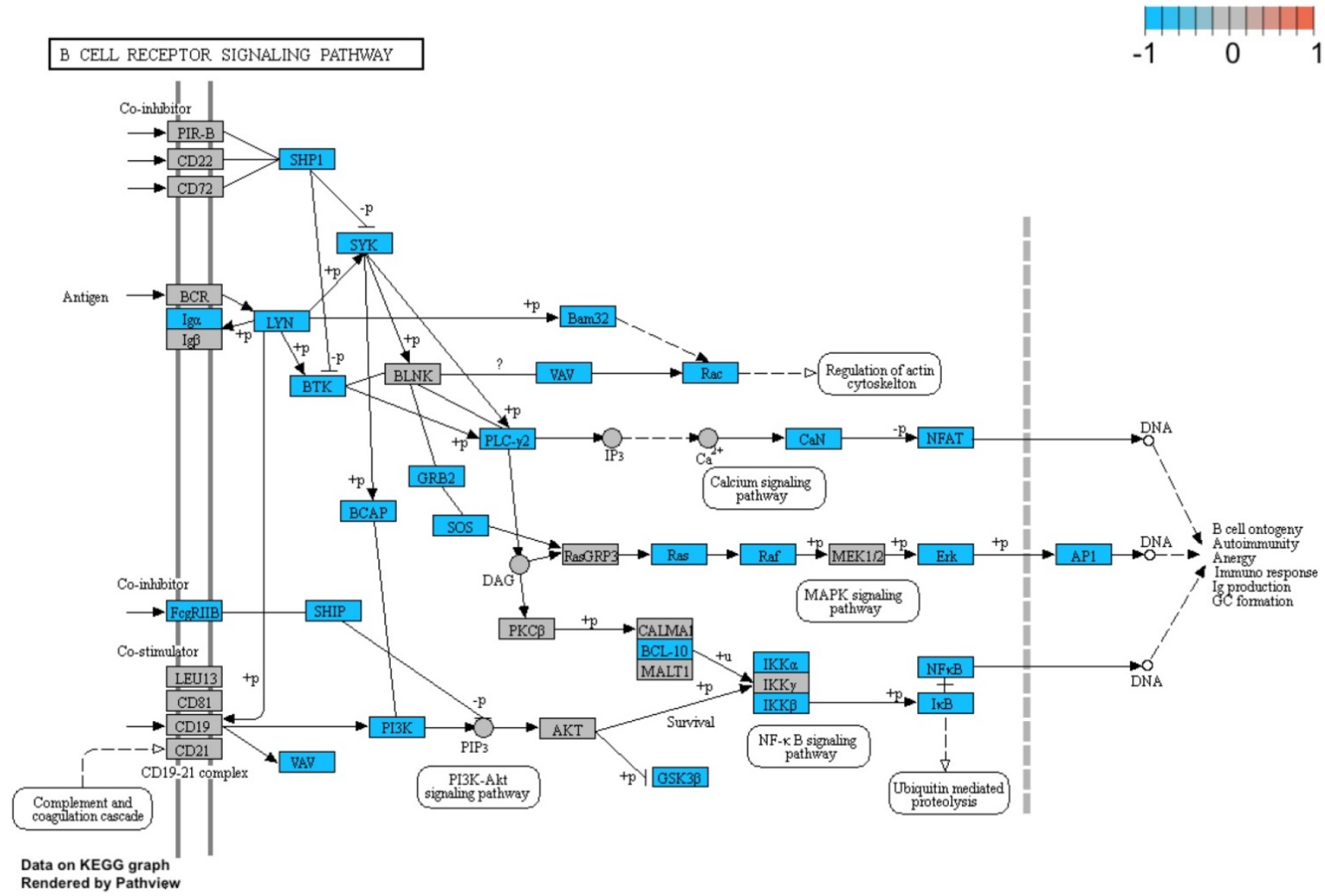

Figure 7. Genes and processes involved in the $04662 . \mathrm{B}$ cell receptor signalling pathway. The colour scale corresponds to log(FC) value, where $\mathrm{FC}$ stands for gene expression fold change when comparing samples from preterm to term neonates. 
expression was significantly downregulated in our preterm neonates group (fold $=-4.32$ ), when compared to term neonates. Interestingly, several studies indicate that $\mathrm{C} 3 \mathrm{a}$ could also have an anti-inflammatory and a neuroprotective effect in the brain tissue against neonatal hypoxia-ischemia injury $(38,39)$. It could indicate that in our preterm neonates group increased C3 levels might serve as a support to immature brain, rather than infection-response signal, but that remains to be further elucidated. In 1997, Wolach et al. once again confirmed lower levels of complement components in preterm neonates and added that C8 and C9 were two complement components that were the most markedly reduced at all gestational ages (40). Our study indicates low levels of C9 in preterm neonates in comparison with term babies. The C9 protein is an essential part of membrane attack complex (MAC), which induces pore formation on a cell membrane and thus causes cell lysis (e.g. bacterial lysis) (41). The C9 deficiency in neonatal serum has been associated with poor defences against bacterial pathogens (33), with premature infants having particularly low C9 levels at even higher risk of bacterial infection (42). Our results of positive correlation between complement components and gestational age suggest gradual development of this system and are in line with previous studies which show that concentrations of complement components gradually increase after birth and reach adult values in 6-18 months of age (43). Nevertheless, the dysregulation of complement in early postnatal days puts neonates at high risk of infections. This might be even more dangerous for preterm babies that are often treated with more invasive procedures that disrupt immunity barriers.

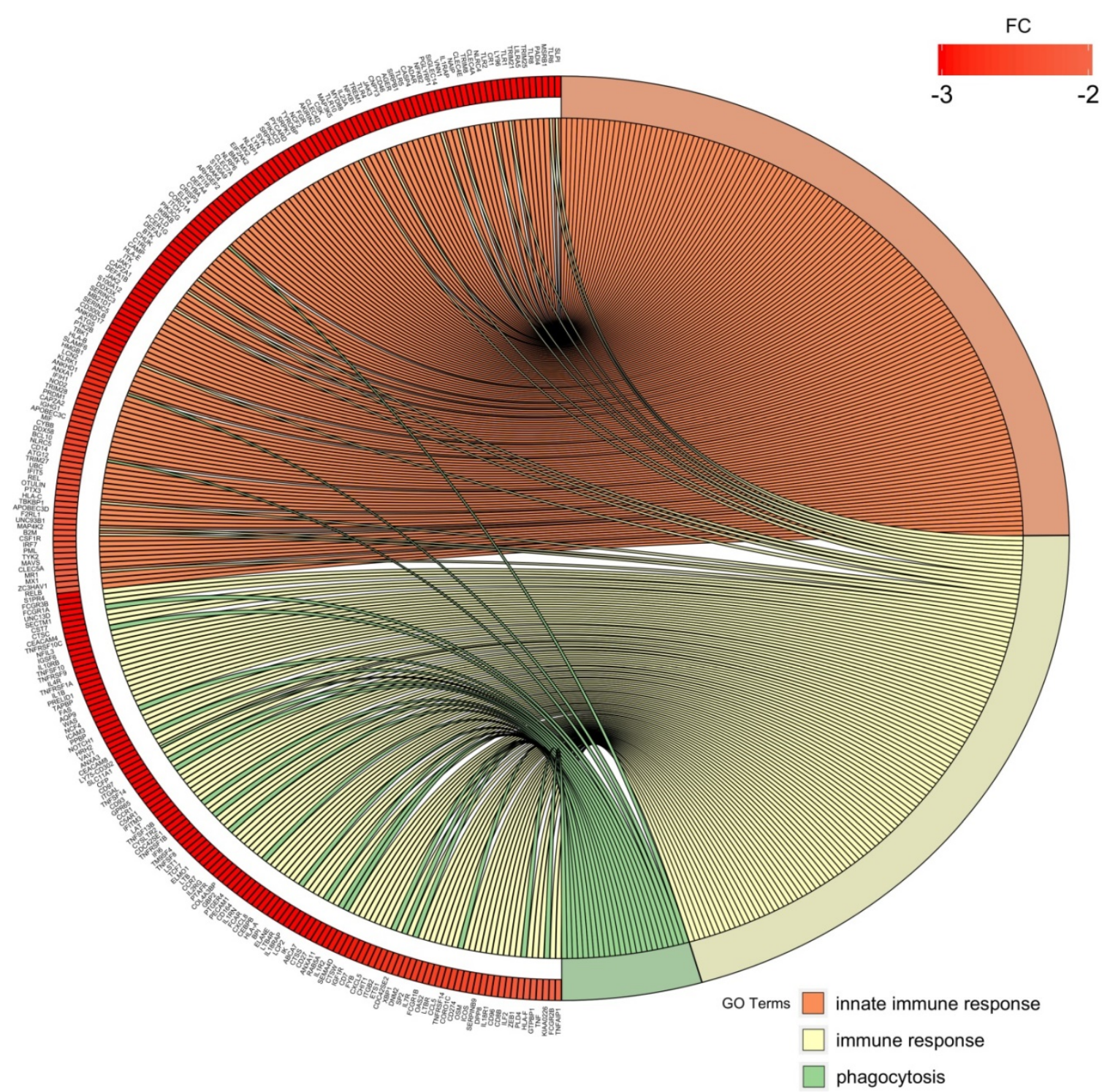

Figure 8. Circos plot shows the dysregulated processes (innate immune response, immune response, phagocytosis) and their associated genes in preterm neonates when compared with term infants. The level of expression for each listed gene is marked using colour scale corresponding to fold change (red = lower expression). Gene involvement in the GO terms is depicted with connecting lines and the ribbons which connect areas of the circos plots indicate also shared genes between groups. 
Several other studies focused on gene expression changes in maternal whole blood, or in amniotic fluid supernatant $(44,45)$. To date, only scarce reports on proteomic and transcriptomic profile of UCB from preterm and term neonates exist. In a recent study, Vora et al. described in UCB samples from preterm babies higher expression of genes involved in cell cycle, which, as authors suggest, might indicate focus on growth and development (46). Our study provided rather contrary results, as we observed downregulation in genes involved in cell cycle, cell division, signal transduction and DNA-templated transcription in preterm neonates in comparison with term babies. This might be attributed to different ethnic groups tested, as in our study all neonates were Caucasian, whereas in the study by Vora et al. both African American and non-Hispanic Whites infants were included. Nevertheless, our results regarding downregulation of genes involved in immune/inflammatory signalling function in preterm infants compared with term infants are in concordance with previously discussed study by Vora et al. and other reports $(47,48)$. In a recent meta-analysis, when eight various foeto-maternal tissue types were analysed separately, only UCB showed significant differentially expressed genes and those UCB samples that came from PTB showed downregulation in several innate immune-related pathways when compared with term samples (47). Interestingly, when the team compared dysregulated genes from UCB analysis with gene expression data from maternal blood meta-analysis, they found 13 genes including toll-like receptor 5 (TLR5) and other transcripts with proven involvement in immune-related processes, which were overlapping and significant in both analyses. The expression pattern of these genes involved in immune response was opposite in UCB samples (downregulation) and maternal whole blood (upregulation) in PTB group when compared with full-term infants.

Toll-like receptors (TLRs) are highly conserved evolutionary receptors of the innate immunity, which constitute the first barrier against pathogens (49-51). In our study we found significantly lower expression of not only TLR5, which confirms previous studies, but also TLR1, 2, 4, 6, 8 and TLR10 were downregulated in our preterm group, when compared with term babies. TLRs have been shown to trigger pro-inflammatory and pro-labour mediators release in uterine epithelial cells, foetal membranes and placenta, which could lead to preterm birth (52). Non-functional protein that is encoded due to nucleotide variants in TLR5, associated with development of bronchopulmonary dysplasia in preterm neonates (53), has also been linked with deficient immune response to flagellated bacteria (47). One of the most downregulated genes in our study, FFAR2, also plays a significant role in immune response to bacteria and specifically gut microbeshost crosstalk, as Ffar2 signalling modulates gut inflammatory tone and pathogen defence (54). In our study we also observed sets of genes tightly related to pathogen-stimulated response to be evidently downregulated, including genes involved in positive regulation of phagocytosis and Fc-gamma receptor signalling pathway involved in phagocytosis. The data so far on impairments in phagocytosis related to prematurity are rather inconsistent $(14,55)$. Recently, Posser et al. reported that phagocytosis in preterm infants is not deficient but rather preterm neonates have fewer phagocytes than term babies (56), which could be a probable factor that contributes to their vulnerability to bacterial infection.

Our results implicate that not only genes involved in innate immunity are highly downregulated in preterm infants, but also expression of those related to the adaptive immunity is lower than in term neonates. Moreover, among those under-expressed genes in our preterm group are also well-known players in multiple biological processes: JAK kinases, NOD2, MAPK kinases family, TLRs, NF-kB family and other, which may suggest inefficiencies in basic cellular processes. The deficiencies in adaptive immunity are rather understandable since adaptive immunity requires acquisition of immunological memory. Previous reports indicate that when compared to adults, neonates, and those born prematurely even more prominently, have generally lower absolute numbers of circulating lymphocytes, deficient $\mathrm{T}$ cell function because of more naïve T cells and less memory $\mathrm{T}$ cells, bias towards Th2 CD4+ $\mathrm{T}$ cell phenotype, reduced production of cytokines such as IFN- $\gamma$, TNF- $\alpha$, IL-12 and lower production $\operatorname{IgG}$ and $\operatorname{IgA}$ antibodies $(57,58)$. It has been suggested that during early days of life significant changes in cell composition and gene expression occur and presumably during this extrauterine 'adaptation time' preterm babies catch up with full-term neonates (59). However, apparently before that happens they are particularly vulnerable, especially due to their deficient immune defense.

\section{Study limitations}

This study provides interesting findings, but some limitations are present. The main limitation is unequally sized groups, which occurred due to difficulties in recruiting preterm neonates to the study. This is quite understandable though, as parents of premature infants are more reluctant to agree on their neonate participation than parents of full-term 
babies. Additionally, in this study women were not tested for antiphosholipid antibodies (positivity is associated with preterm birth), as in Poland this testing is recommended only in recurrent miscarriages. Another possible limitation of this study is the borderline significance of difference in IL-6 levels between tested groups, which could reflect non-clinically evident infections.

\section{Conclusions}

Prematurely born neonates are at high risk of immediate and long-term complications following preterm birth. Disturbances in immune functions have been suggested to contribute to both preterm birth and prematurity-related complications. Our results indicate differences in complement components concentration and a significant downregulation of over 3,000 genes in UCB from preterm infants when compared with term babies. These differentially expressed genes are involved mainly in various immune-related pathways, including innate immune response, phagocytosis and TLR function. Further functional studies are required to elucidate how this gene downregulation affects pathways that they are involved in. Our findings emphasize the need for further studies on large cohorts for better understanding of postnatal stages in development of immune system.

\section{Acknowledgements}

This work was supported by Pomeranian Medical University statutory funds.

\section{Competing Interests}

The authors have declared that no competing interest exists.

\section{References}

1. Frey HA, Klebanoff MA. The epidemiology, etiology, and costs of preterm birth. Semin Fetal Neonatal Med. 2016;21(2):68-73.

2. Liu L, Oza S, Hogan D, Chu Y, Perin J, Zhu J, et al. Global, regional, and national causes of under-5 mortality in 2000-15: an updated systematic analysis with implications for the Sustainable Development Goals. Lancet. 2016;388(10063):3027-35.

3. Glass HC, Costarino AT, Stayer SA, Brett CM, Cladis F, Davis PJ. Outcomes for extremely premature infants. Anesthesia and analgesia. 2015;120(6):1337-51.

4. Srinivasjois R, Slimings C, Einarsdóttir K, Burgner D, Leonard H. Association of Gestational Age at Birth with Reasons for Subsequent Hospitalisation: 18 Years of Follow-Up in a Western Australian Population Study. PloS one. 2015;10(6):e0130535-e.

5. de Jong M, Verhoeven M, van Baar AL. School outcome, cognitive functioning, and behaviour problems in moderate and late preterm children and adults: a review. Semin Fetal Neonatal Med. 2012;17(3):163-9.

6. Lee KJ, Yoo J, Kim YH, Kim SH, Kim SC, Kwak DW, et al. The Clinical Usefulness of Predictive Models for Preterm Birth with Potential Benefits: A KOrean Preterm collaboratE Network (KOPEN) Registry-Linked Data-Based Cohort Study. Int J Med Sci. 2020;17(1):1-12.

7. Koullali B, Oudijk MA, Nijman TA, Mol BW, Pajkrt E. Risk assessment and management to prevent preterm birth. Semin Fetal Neonatal Med. 2016;21(2):80-8.

8. Kemp MW. Preterm birth, intrauterine infection, and fetal inflammation. Frontiers in immunology. 2014;5:574-.

9. Kanellopoulos-Langevin C, Caucheteux SM, Verbeke P, Ojcius DM. Tolerance of the fetus by the maternal immune system: role of inflammatory mediators at the feto-maternal interface. Reproductive biology and endocrinology RB\&E. 2003;1:121

10. Gomez-Lopez N, StLouis D, Lehr MA, Sanchez-Rodriguez EN, Arenas-Hernandez M. Immune cells in term and preterm labor. Cell Mol Immunol. 2014;11(6):571-81.

11. Romero R, Dey SK, Fisher SJ. Preterm labor: one syndrome, many causes. Science (New York, NY). 2014;345(6198):760-5.

12. Cappelletti M, Della Bella S, Ferrazzi E, Mavilio D, Divanovic S. Inflammation and preterm birth. J Leukoc Biol. 2016;99(1):67-78.

13. Sharma AA, Jen R, Butler A, Lavoie PM. The developing human preterm neonatal immune system: a case for more research in this area. Clinical immunology (Orlando, Fla). 2012;145(1):61-8.

14. Helmo FR, Alves EAR, Moreira RAA, Severino VO, Rocha LP, Monteiro M, et al. Intrauterine infection, immune system and premature birth. J Matern Fetal Neonatal Med. 2018;31(9):1227-33.

15. Melville JM, Moss TJ. The immune consequences of preterm birth. Front Neurosci. 2013;7:79.

16. Simon AK, Hollander GA, McMichael A. Evolution of the immune system in humans from infancy to old age. Proceedings Biological sciences. 2015;282(1821):20143085-

17. Niewiesk S. Maternal antibodies: clinical significance, mechanism of interference with immune responses, and possible vaccination strategies. Frontiers in immunology. 2014;5:446-.

18. Galindo-Sevilla N, Reyes-Arroyo F, Mancilla-Ramirez J. The role of complement in preterm birth and prematurity. J Perinat Med. 2019;47(8):793-803.

19. Sarma JV, Ward PA. The complement system. Cell and tissue research. 2011;343(1):227-35

20. McGreal EP, Hearne K, Spiller OB. Off to a slow start: under-development of the complement system in term newborns is more substantial following premature birth. Immunobiology. 2012;217(2):176-86.

21. Gródecka-Szwajkiewicz D, Ulańczyk Z, Zagrodnik E, Łuczkowska K, Rogińska D, Kawa MP, et al. Differential Secretion of Angiopoietic Factors and Expression of MicroRNA in Umbilical Cord Blood from Healthy Appropriate-For-Gestational-Age Preterm and Term Newborns - in Search of Biomarkers of Angiogenesis-Related Processes in Preterm Birth . International Journal of Molecular Sciences. 2020;21(4):1305.

22. NETCORD-FACT. Sixth Edition NetCord-FACT International Standards for Cord Blood Collection, Banking, and Release for Administration.2016.

23. Rudnicki J, Kawa MP, Kotowski M, Michalczyk B, Ustianowski P, Czajka R, et al. Clinical Evaluation of the Safety and Feasibility of Whole Autologous Cord Blood Transplant as a Source of Stem and Progenitor Cells for Extremely Premature Neonates: Preliminary Report. Exp Clin Transplant. 2015;13(6):563-72.

24. Cymbaluk-Ploska A, Chudecka-Glaz A, Pius-Sadowska E, Sompolska-Rzechula A, Chudecka K, Bulsa M, et al. Clinical Relevance of NGAL/MMP-9 Pathway in Patients with Endometrial Cancer. Dis Markers. 2017;2017:6589262.

25. Luczkowska K, Roginska D, Ulanczyk Z, Machalinski B. Effect of Bortezomib on Global Gene Expression in PC12-Derived Nerve Cells. Int J Mol Sci. 2020;21(3).

26. Luczkowska K, Roginska D, Ulanczyk Z, Paczkowska E, Schmidt CA, Machalinski B. Molecular Mechanisms of Bortezomib Action: Novel Evidence for the miRNA-mRNA Interaction Involvement. Int J Mol Sci. 2020;21(1).

27. Benjamini $Y$, Hochberg Y. Controlling the False Discovery Rate: A Practical and Powerful Approach to Multiple Testing. Journal of the Royal Statistical Society Series B (Methodological). 1995;57(1):289-300.

28. Regal JF, Gilbert JS, Burwick RM. The complement system and adverse pregnancy outcomes. Molecular immunology. 2015;67(1):56-70.

29. Drew JH, Arroyave CM. The complement system of the newborn infant. Biol Neonate. 1980;37(3-4):209-17.

30. Grumach AS, Ceccon ME, Rutz R, Fertig A, Kirschfink M. Complement profile in neonates of different gestational ages. Scand J Immunol. 2014;79(4):276-81.

31. Notarangelo LD, Chirico G, Chiara A, Colombo A, Rondini G, Plebani A, et al. Activity of classical and alternative pathways of complement in preterm and small for gestational age infants. Pediatr Res. 1984;18(3):281-5.

32. Maródi L. Neonatal innate immunity to infectious agents. Infection and immunity. 2006;74(4):1999-2006.

33. Wynn JL, Levy O. Role of innate host defenses in susceptibility to early-onset neonatal sepsis. Clinics in perinatology. 2010;37(2):307-37.

34. Georgountzou A, Papadopoulos NG. Postnatal Innate Immune Development: From Birth to Adulthood. Frontiers in immunology. 2017;8:957-

35. Lupu F, Keshari RS, Lambris JD, Coggeshall KM. Crosstalk between the coagulation and complement systems in sepsis. Thrombosis research. 2014;133 Suppl 1(0 1):S28-S31.

36. Zilow EP, Hauck W, Linderkamp O, Zilow G. Alternative pathway activation of the complement system in preterm infants with early onset infection. Pediatr Res. 1997;41(3):334-9.

37. Strunk T, Currie A, Richmond P, Simmer K, Burgner D. Innate immunity in human newborn infants: prematurity means more than immaturity. J Matern Fetal Neonatal Med. 2011;24(1):25-31.

38. Shim YJ, Choi BY, Park KH, Lee H, Jung YM, Kim YM. Inflammatory and Immune Proteins in Umbilical Cord Blood: Association with Hearing Screening Test Failure in Preterm Neonates. Mediators of inflammation. 2018;2018:4209359-. 
39. Kildsgaard J, Hollmann TJ, Matthews KW, Bian K, Murad F, Wetsel RA. Cutting edge: targeted disruption of the $\mathrm{C} 3 \mathrm{a}$ receptor gene demonstrates a novel protective anti-inflammatory role for C3a in endotoxin-shock. J Immunol. 2000;165(10):5406-9.

40. Wolach B, Dolfin T, Regev R, Gilboa S, Schlesinger M. The development of the complement system after 28 weeks' gestation. Acta Paediatrica. 1997;86(5):523-7.

41. Bayly-Jones C, Bubeck D, Dunstone MA. The mystery behind membrane insertion: a review of the complement membrane attack complex. Philosophical transactions of the Royal Society of London Series B, Biological sciences. 2017;372(1726):20160221.

42. Lassiter HA, Watson SW, Seifring ML, Tanner JE. Complement factor 9 deficiency in serum of human neonates. J Infect Dis. 1992;166(1):53-7.

43. Davis CA, Vallota EH, Forristal J. Serum complement levels in infancy: age related changes. Pediatr Res. 1979;13(9):1043-6.

44. Zwemer LM, Bianchi DW. The amniotic fluid transcriptome as a guide to understanding fetal disease. Cold Spring Harbor perspectives in medicine. 2015;5(4):a023101.

45. Paquette AG, Shynlova O, Kibschull M, Price ND, Lye SJ. Comparative analysis of gene expression in maternal peripheral blood and monocytes during spontaneous preterm labor. Am J Obstet Gynecol. 2018;218(3):345.e1-.e30

46. Vora NL, Parker JS, Mieckowski PA, Smeester L, Fry RC, Boggess KA. RNA-Sequencing of Umbilical Cord Blood to Investigate Spontaneous Preterm Birth: A Pilot Study. AJP reports. 2019;9(1):e60-e6.

47. Vora B, Wang A, Kosti I, Huang H, Paranjpe I, Woodruff TJ, et al. Meta-Analysis of Maternal and Fetal Transcriptomic Data Elucidates the Role of Adaptive and Innate Immunity in Preterm Birth. Front Immunol. 2018;9:993.

48. Hilgendorff A, Windhorst A, Klein M, Tchatalbachev S, Windemuth-Kieselbach C, Kreuder I, et al. Gene expression profiling at birth characterizing the preterm infant with early onset infection. Journal of molecular medicine (Berlin, Germany). 2017;95(2):169-80.

49. Parker LC, Prince LR, Sabroe I. Translational mini-review series on Toll-like receptors: networks regulated by Toll-like receptors mediate innate and adaptive immunity. Clinical and experimental immunology. 2007;147(2):199-207.

50. Liu J, Chen T, Lei P, Tang X, Huang P. Exosomes Released by Bone Marrow Mesenchymal Stem Cells Attenuate Lung Injury Induced by Intestinal Ischemia Reperfusion via the TLR4/NF-kappaB Pathway. Int J Med Sci. 2019;16(9):1238-44.

51. Chan JYW, Tsui JCC, Law PTW, So WKW, Leung DYP, Sham MMK, et al. Regulation of TLR4 in silica-induced inflammation: An underlying mechanism of silicosis. Int J Med Sci. 2018;15(10):986-91.

52. Lim R, Barker G, Lappas M. TLR2, TLR3 and TLR5 regulation of pro-inflammatory and pro-labour mediators in human primary myometrial cells. J Reprod Immunol. 2017;122:28-36.

53. Sampath V, Garland JS, Le M, Patel AL, Konduri GG, Cohen JD, et al. A TLR5 (g.1174C > T) variant that encodes a stop codon (R392X) is associated with bronchopulmonary dysplasia. Pediatr Pulmonol. 2012;47(5):460-8.

54. Chun E, Lavoie S, Fonseca-Pereira D, Bae S, Michaud M, Hoveyda HR, et al. Metabolite-Sensing Receptor Ffar2 Regulates Colonic Group 3 Innate Lymphoid Cells and Gut Immunity. Immunity. 2019;51(5):871-84.e6.

55. Källman J, Schollin J, Schalèn C, Erlandsson A, Kihlström E. Impaired phagocytosis and opsonisation towards group B streptococci in preterm neonates. Archives of disease in childhood Fetal and neonatal edition. 1998;78(1):F46-F50.

56. Prosser A, Hibbert J, Strunk $\mathrm{T}$, Kok $\mathrm{CH}$, Simmer $\mathrm{K}$, Richmond $\mathrm{P}$, et al Phagocytosis of neonatal pathogens by peripheral blood neutrophils and monocytes from newborn preterm and term infants. Pediatr Res. 2013;74(5):503-10.

57. Goronzy JJ, Fang F, Cavanagh MM, Qi Q, Weyand CM. Naive T cell maintenance and function in human aging. Journal of immunology (Baltimore, Md : 1950). 2015;194(9):4073-80.

58. Yu JC, Khodadadi H, Malik A, Davidson B, Salles ÉdSL, Bhatia J, et al. Innate Immunity of Neonates and Infants. Frontiers in immunology. 2018;9:1759-

59. Olin A, Henckel E, Chen Y, Lakshmikanth T, Pou C, Mikes J, et al. Stereotypic Immune System Development in Newborn Children. Cell. 2018;174(5):1277-92.e14. 